\title{
Microneedles: a therapeutic alternative in melasma
}

\begin{abstract}
Background: Melasma is an acquired hypermelanosis of the skin, which compromises the photo-exposed areas of the face. It mainly affects women and the darker photo types (Photo types of Fitzpatrick III-V), causes a great impact on the quality of life of patients who suffer from it, being of concern the therapeutic options available. Micro needles are a novel adjuvant therapeutic modality, their procedure is simple, well tolerated and with cosmetic benefits.
\end{abstract}

Objective: To conduct a review of medical literature related to micro needles for the treatment of melasma

Method: A literature search was performed using the PubMed, Medline and Embase databases. Additionally, the bibliography of relevant articles was reviewed.
Volume 2 Issue 4 - 2018

\author{
Claudia Marcela Arenas Soto \\ Department of Dermatology, Universidad Militar Nueva \\ Granada Bogota, Colombia
}

Correspondence: Claudia Marcela Arenas Soto, Department of Dermatology, Military Central Hospital, Universidad Militar Nueva Granada Bogota, Colombia, Email draclaudiaarenas@gmail.com

Received: May 17, 2018 | Published: August 23, 2018

Keywords: melanosis, pigmentation, microneedles, collagen

\section{Introduction}

Melasma is a dysfunction of melanogenesis, which produces symmetrical hyperpigmented macules in the skin's exposed areas, prevalence has been estimated in $1 \%$ of the general population and is higher in at-risk populations, its pathogenesis is not clear, but have described some triggers such as ultraviolet rays (UVR), pregnancy, sex hormones, inflammatory skin processes, the use of cosmetics, steroids and photosensitizing drugs, family history is also considered an important risk factor. ${ }^{1}$ These factors trigger different signaling pathways such as those dependent on the melanocyte stimulating hormone that induces cyclic adenosine monophosphate (MSH/cAMP), also the tyrosine kinase receptor (KIT) and the Wnt transduction pathway, which regulate positive tyrosinase and transcription factor associated with microphthalmia (MITF), which results in the stimulation of melanogenesis and the development of melasma. $^{2}$

The most usual presentation is located in areas of the forehead, nose and upper lip, respecting the areas that are not exposed to the sun. Histologically, it may show greater epidermal or dermal pigmentation, increased melanocytes, increased melanosomes, solar elastosis, and occasionally perivascular lymphohistiocytic infiltrates. ${ }^{3}$ The treatment of melasma has become a great challenge that seeks to improve the quality of life of patients who suffer from it; within the therapeutic alternatives arises the microneedle technique, it is a minimally invasive, safe, well tolerated and effective procedure.

\section{Discussion}

The main concept dates from 23years ago with Orentreich and Orentreich who described the management of scars and wrinkles with intradermal needles. ${ }^{4}$ Later in 1997 Camirand and Doucet described a technique with tattoo guns to improve the appearance of postsurgical scars by releasing tension. ${ }^{5}$ The first micro needle device was developed in 2000 for rejuvenation and in 2003 Fernandes developed a cylindrical device with multiple needles. ${ }^{6}$

\section{Mechanism of action}

The normal human epidermis has a transepithelial potential (PET) that varies in different parts of the body $(10-60 \mathrm{mV})$. In human skin, the transepithelial potential (PET) is measurable and is established mainly after the asymmetric distribution of sodium ions $\left(\mathrm{Na}^{+}\right)$in the lower and upper sections of the epidermis. In the normal human epidermis, the measured values of the transepithelial potential are between 10 and $60 \mathrm{mV}$ and vary according to the anatomical location. The role of PET in the normal epidermis has not yet been identified; but after a skin injury, disruption of PET induces an endogenous continuous electric current field $(100-200 \mathrm{mV} / \mathrm{mm})$ directed towards the center of the wound. This endogenous electric field could be involved in the process of wound healing by attracting cells, which would facilitate re-epithelialization. Increasing and decreasing TEP values correlate with changes in the expression of the potassium sodium pump $\left(\mathrm{Na}^{+} /\right.$ $\mathrm{K}^{+}$ATPase). The distribution of $\mathrm{Na}^{+} / \mathrm{K}^{+}$ATPase pumps also varies according to epidermal differentiation. ${ }^{7}$

The micro needle technique or also called collagen-inducing therapy produces a controlled lesion of the skin, these micro-lesions trigger a cascade of healing that starts generating electrical impulses, which are caused by the modification of the transepithelial action potential. The micro needles when penetrating the stratum corneum generate a short circuit of the endogenous electric fields, this activates the $\mathrm{Na}^{+} / \mathrm{K}^{+}$pump seeking to reestablish the inter and intracellular electrical potential caused by the injury; subsequently the cells that surround the channels produced by the needles, detect these repeated penetration stimuli, creating a polarized electromagnetic field, which stimulates the DNA to generate gene expression of proteins from the surrounding cells, with consequent migration of epithelial cells, endothelial cells and releases growth factors such as: platelet-derived growth factor (PGF), transforming growth factor alpha and beta (TGF- $\alpha$ and TGF- $\beta$ ), connective tissue activating protein, connective tissue growth factor and growth of fibroblasts (FGF) (Figure 1). The migration, proliferation and fixation of fibroblasts to the intercellular matrix initiate neocolagenesis and neovascularization, this matrix arrangement allows the deposition of type III collagen and eventually its replacement by type I collagen. ${ }^{7-10}$

The most frequent adverse effects of the technique consist of edema, erythema, and solar sensitivity. In a lesser proportion, pain may occur during the procedure, bruising and irritation. ${ }^{12}$ Few cases of post inflammatory hyper pigmentation have been reported. ${ }^{13,14}$ The formation of scars has been related to the length of the needles on 
surfaces in contact with bony prominences. ${ }^{15}$ No study has reported cases of bacterial super infection, the use of sterile elements and disinfection prior to the procedure is sufficient to avoid it. Some studies have reported infections with herpes simplex virus, which were successfully treated with acyclovir. ${ }^{16,17}$ (Figure 1) (Figure 2) (Table 1)

$\begin{array}{lll}\begin{array}{l}\text { Microneedle } \\ \text { devices }\end{array} & \text { Rollers manuales } & \text { Electrical devices with micro needles }\end{array}$

\section{Definition}

Types

Advantage
Manual device with cylindrical roller with 24 circular dies. Each matrix is equipped with 8 steel micro needles, in total 192 needles (Fig2A).
Automated microneedle device similar to a ballpoint pen. Use disposable needles and guides; adjust according to the length of the needle (Fig2B).

Dermapen: The tip contains 9-12 needles arranged in rows. Presents rechargeable battery that operates with 2 modes (high speed 700 cycles / $\mathrm{min}$ and low speed $4 / 2$ cycles / $\mathrm{min}$ ). - DermaFrac: Combines microdermabrasion with micro needles, simultaneous infusion of topical management to deep tissue and light emitting diode therapy. For treatment of skin with actinic damage, acne, post inflammatory hyper pigmentation, wrinkles, fine lines, hyper pigmentation and superficial scars
MS-4: smaller cylinder formed by 4 circular needle arrays (in total 96 needles) that have a length of $1.5 \mathrm{~mm}$. For areas that require greater precision and deeper penetration.

-The Beauty Mouse: approved for cosmetic use contains 480 needles of approximately $0.2 \mathrm{~mm}$. For use on large skin surfaces

$\mathrm{C} 8, \mathrm{C} 8-\mathrm{HE}$ and Beauty Mouse cosmetic use, per patient.

- Economic

- Treatment of large areas
Ability to adjust speed and penetration depth.

- Treatment of large areas

- Disposable needle tips that limit the risk of infection

- Access to small and inaccessible areas such as periorifial areas

- Less cutaneous trauma

- Economic

Non-disposable needles

- Risk of infection

- Variable penetration speed and depth.

- Increased skin trauma

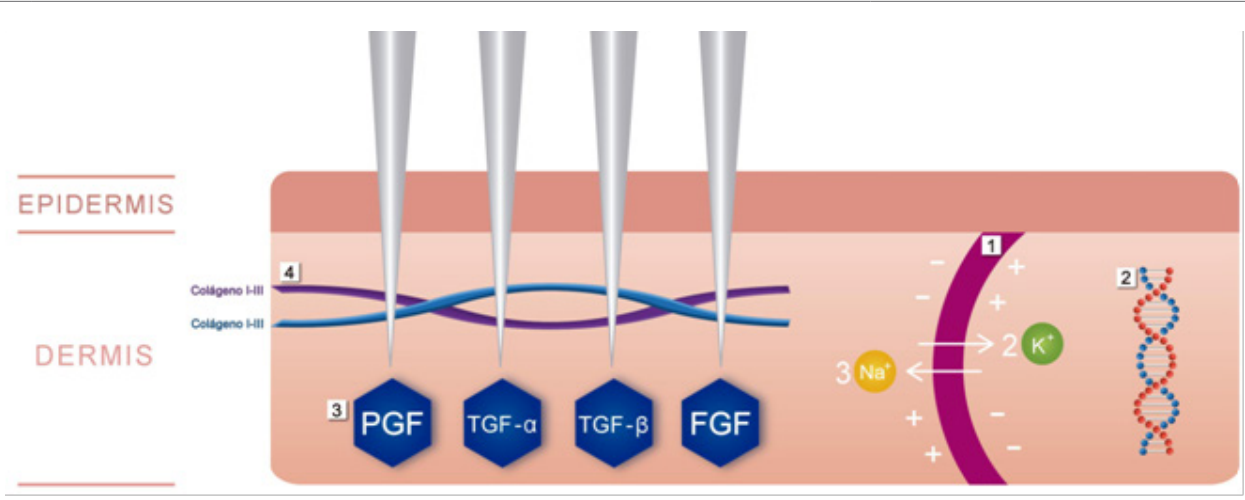

Figure I Mechanism of action of the microneedles.

i. When the microlesion is generated, a short circuit occurs in the PET that activates the Na+/ K/ ATPase Pumps.

ii. The repeated stimulus creates an electromagnetic field that induces the DNA of the adjacent cells to produce chemotactic factors

iii. Migration occurs of cells and growth factors

iv. Collagen type III is deposited that can be replaced eventually by collagen type I. 


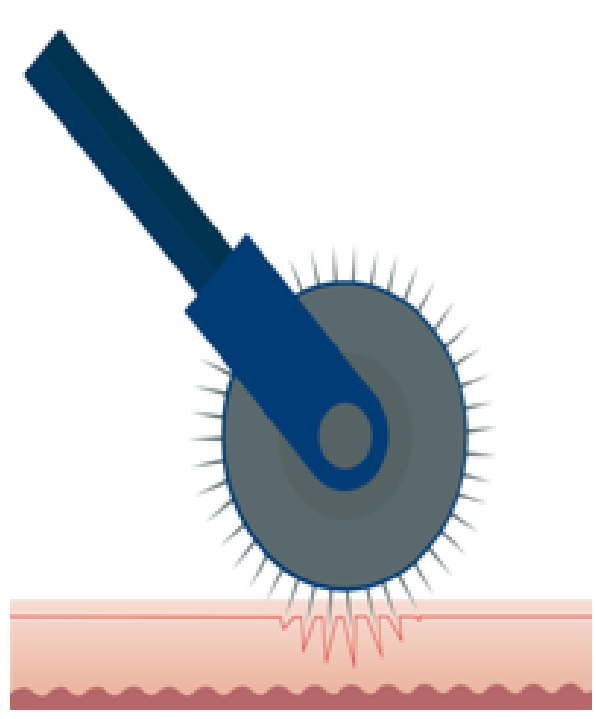

Figure 2A Dermaroller device.

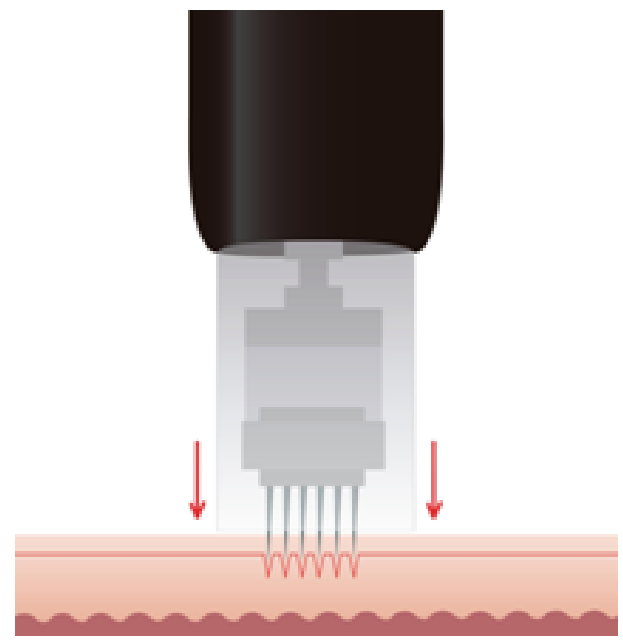

Figure 2B Dispositivo Dermapen.

\section{Use in melasma and melanogenesis}

The micro needles began to be used for the treatment of acne scars and it was observed that in patients with concomitant melasma, there was an improvement in the decrease of the pigment in the skin. ${ }^{18,19} \mathrm{In}$ this way, it was begun to be explored as a means of transdermal drug administration in a population with Fitzpatrick III-IV-V phototype, for the treatment of hyper pigmentation disorders. ${ }^{20}$ The long-term improvement of recalcitrant melasma after micro needle treatment has been reported in case series studies, however, the exact mechanism that promotes skin clearance is unknown. ${ }^{18,19}$

In a small study conducted in patients from the northeast of Brazil by Lima and colleagues, after the performance of 2 sessions with micro needles subjective improvement of the skin smoothness and greater luminosity in patients with recalcitrant melasma was observed. A decrease of $70 \%$ in the MASI, $13 \%$ increase in luminosity and $55 \%$ decrease in the melasma quality of life scale was evidenced. In the histological findings, it was considered that micro needle therapy promotes the proliferation of fibroblasts and superior dermal neocogenesis, decreases the contact of the melanocytes with melanogenic stimuli and improves the protection against ultraviolet radiation due to epidermal thickening. ${ }^{18}$

Lima et al reported effectiveness of micro needle treatment in 22 cases of patients with recalcitrant melasma, followed 2days after the application of depigmentation therapy and sun protection, with maintenance of clearance at 2 and 11 months of follow-up. The author considers that the trauma caused should be mild and the use of depigmenting and sunscreen should be mandatory. ${ }^{19}$ Budamakunkla and colleagues observed better results in 60patients with moderate to severe melasma treated with micro needles plus the application of topical tranexamic acid compared with microinjections. ${ }^{20,21,8}$ Fabbrocini et al. Demonstrated in a pilot study of 20patients diagnosed with melasma that the combination therapy of micro needles with topical depigmenting agents is more effective than monotherapy with topical depigmenting agents. ${ }^{8,20,21}$,

\section{Conclusion}

The inducer therapy of collagen with micro needles in hyper pigmentation processes constitutes a simple, economic, well tolerated, effective, novel adjuvant therapy, with long-term cosmetic benefits and few adverse effects. It is a procedure that stands out for the epidermal preservation, in addition to stimulating the production of collagen and elastin at the dermal level, which contributes to the decrease of the visible pigment. Thanks to the formation of micro channels can be used in conjunction with topical depigmenting improving its absorption and effectiveness.

\section{Acknowledgements}

None.

\section{Conflict of interest}

Author declares that there is no conflict of interest.

\section{References}

1. Handel AC, Bartoli LD, Miot HA. Melasma: a clinical and epidemiological review. An Bras Dermatol. 2014;89(5):771-782.

2. Lee AY. Recent progress in melasma pathogenesis. Pigment Cell Melanoma Res. 2015;28(6):648-660.

3. Ogbechie-Godec OA, Elbuluk N. Melasma: An Up-to-Date Comprehensive Review. Dermatol Ther (Heidelb). 2017;7(3):305-318.

4. Orentreich DS, Orentreich N. Subcutaneous incisionless (subcision) surgery for the correction of depressed scars and wrinkles. Dermatol Surg. 1995;21(6):543-549.

5. Camirand A, Doucet J. Needle dermabrasion. Aesthet Plast Surg. 1997;21(1):48-51.

6. Fernandes D. Minimally invasive percutaneous collagen induction. Oral Maxillofac Surg Clin North Am. 2005;17(1):51-63.

7. Dube J, Rochette-Drouin O, Lévesque P, et al. Restoration of the transepithelial potential Within Tissue-Engineered Human Skin In Vitro and During the Wound Healing Process in vivo. Tissue Eng Part A. 2010;16(10):3055-3063.

8. Singh A, Yadav S. Microneedling: Advances and widening horizons. Indian Dermatol Online J. 2016;7(4):244-254.

9. Konicke K, Knabel M, Olasz E. Microneedling: A Primer for Dermatologists. J Dermatol Plast Surg. 2017;2(2):1014. 
10. Liebl H, Kloth LC. Skin Cell Proliferation Stimulation by Microneedles. J Am Coll Clin Wound Spec. 2012;4(1):2-6.

11. Alster TS, Graham PM. Microneedling: A Review and Practical Guide. Dermatol Surg. 2018;44(3):397-404.

12. Iriarte $\mathrm{C}$, Awosika O, Rengifo-Pardo M, et al. Review of applications of microneedling in dermatology. Clin Cosmet Investig Dermatol. 2017:10:289-298.

13. Ramaut L, Hoeksema H, Pirayesh A, et al. Microneedling: Where do we stand now? A systematic review of the literature. J Plast Reconstr Aesthet Surg. 2018;71(1):1-14.

14. Asif M, Kanodia S, Singh K. Combined autologous platelet-rich plasma with microneedling versus microneedling with distilled water in the treatment of atrophic acne scars: a concurrent split-face study. J Cosmet Dermatol. 2016;15(4):434-443.

15. Majid I. Microneedling therapy in atrophic facial scars: An objective assessment. J Cutan Aesthet Surg. 2009;2(1);26-30.

16. Pahwa M, Pahwa P, Zaheer A. "Tram track effect" after treatment of acne scars using a microneedling device. Dermatologic Surg. 2012;38(7 Pt 1);1107-1108.
17. Aust MC, Fernandes D, Kolokythas P, et al. Percutaneous collagen induction therapy. An alternative treatment for scars, wrinkles, and skin laxity. Plast Reconstr Surg. 2008;121(4):1421-1429.

18. Lima EVA, Lima MMDA, Paixão MP, et al. Assessment of the effects of skin microneedling as adjuvant therapy for facial melasma: a pilot study. BMC Dermatol. 2017;28;17(1):14.

19. Lima Ede A. Microneedling in facial recalcitrant melasma: report of a series of 22 cases. An Bras Dermatol. 2015;90(6):919-921.

20. Cohen BE, Elbuluk N. Microneedling in skin of color: A review of uses and efficacy. J Am Acad Dermatol. 2016 Feb;74(2):348-355.

21. Hou A, Cohen B, Haimovic A, et al. Microneedling: A Comprehensive Review. Dermatol Surg. 2017;43(3):321-339.

22. Osman-Ponchet H, Alriquet M, Kouidhi M, et al. Use of Microneedle Device to Enhance Dermal Absorption: Study on Ex Vivo Human Skin. J Dermat Cosmetol. 2018;2(1):00032. 